Proceedings of the $\mathbf{2}^{\text {nd }}$ ICEENG Conference, 23-25 Nov 1999

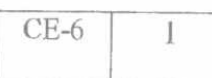

Military Technical College

Kobry Elkobbah,

Cairo, Egypt

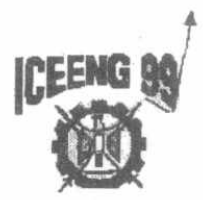

$2^{\text {nd }}$ International Conference on Electrical Engineering

ICEENG 9

\title{
FROM NEURAL NETWORK TO AIRCRAFT RECOGNITION SYSTEM
}

\author{
A. A. Somaie*, A. Badr** and T. Salah*
}

\begin{abstract}
In this paper, an aircraft recognition system using a neural network is presented. A 2-D perspective view of aircraft models is first normalized through the preprocessing stage using bilinear interpolation and principal component analysis. The new patterns are invariant to translation, dilation, and rotation. Then, the Kohonen and Grossberg neural networks were trained using a small number of normalized patterns. The presented algorithm was tested on partially incomplete, noisy and geometrically distorted images and it was found that the recognition performance is $100 \%$ with six referenced aircraft.
\end{abstract}

Key words: Image processing, Neural network, Pattern recognition.

${ }^{*} \mathrm{R} \& \mathrm{D}$ Centre, EAF

Cairo, e-mail: aaisomaia@hotmail.com

** Comp \& Sys Eng. Dep., Faculty of Eng.,

Ain Shams University, Cairo, e-mail: abadr@idsc.gov.eg 
Proceedings of the $\mathbf{2}^{\text {nd }}$ ICEENG Conference, 23-25 Nov 1999

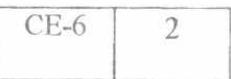

\section{INTRODUCTION}

An important aspect of human visual system is the ability to recognize an object despite changes in the object's shift, scale, and rotation. A machine vision system is the implementation of human visual system. The methods adopted for this purpose can be done, which exploit tactile information to build an analytical model of the explored object. Structural methods describe an object by means of grammar of constituent primitive parts and associated relationships. Neural approaches, which learn from a set of examples the knowledge necessary for object recognition. The first two model-based methods are quite sensitive to the noise affecting sensorial data, and are preferable when dealing with objects that contain an indefinite number of elements. On the contrast, the neural methods could be suitable for recognizing the object on hand.

Tseng et al [1] defined a neural network model for a 3-D recognition system. His approach was robust, since it can recognize the object successfully even, if it was partially viewed or noisily corrupted. Mao et al [2] presented a complete study about architecture of number of networks and learning algorithms, which is used for feature extraction and data projection. Ravichandran and Yegnanarayana [3] proposed the artificial neural network models used for object recognition. These objects were Olympic game symbols. Caselli et al [4] used the geometric properties of a predefined set of very different shapes and sizes for learning, followed by the recognition phase. Both phases are mapped on training and classification by using the unsupervised Kohonen's selforganizing feature map. Perantonis and Lisboa [5] presented a high-order network to solve the classification and recognition of two-dimensional patterns independently of their position, orientation and size.

In this paper, we present an aircraft recognition system using neural network invariant to position, scale, and orientation. The normalized patterns are fed into the aircraft neural model. This stage contains a two-layer network. The first layer, Kohonen's layer, classifies the similar input vectors into distinct groups. The second layer, Grossberg network, is responsible for producing the desired outputs or the recognized target. The normalized image pattern was obtained using the preprocessing stage and this is presented in section II.

The description of the Kohenen and Crossberg models or the Counter Propagation Network (CPN) is defined in section III. The complete aircraft recognition system has been tested using six of aircraft types and some of the experimental results are presented in section $I V$. Section $V$ contains the conclusions.

\section{PREPROCESSING STAGE}

The aircraft image data were taken using a frame transfer to CCD camera model 4712 (625 lines CCIR standard) manufactured by COHU and fitted with a f/1.4 Cosmicar TV lens of $25 \mathrm{~mm}$ focal length for image capturing. The camera was connected to a PIP1024 frame grabber, manufactured by Matrix, which occupied one of the expansion slots of a Pentium $100 \mathrm{MHz}$ PC. All images were digitized to 256 gray levels and were 512 by 512 pixels in size. A 
black curtain was used to provide a background and all objects were painted white. Each aircraft model is rotated $n \pi / 4$ degree $(n=0,1, \ldots \ldots, 7)$ to get different orientation of snapshots. A set of eight snapshots (set1) of each object was taken under nominally the same conditions. Another set called set2 of 10 snapshots was grabbed to each aircraft model under different orientation and lighting circumstances with fixed camera position. Five versions of aircraft images of each type from set 1 were utilized for training the net. The other thirteen versions were used as a test pattern. Fig. 1 shows the images of the aircraft models after aspect ratio correction.
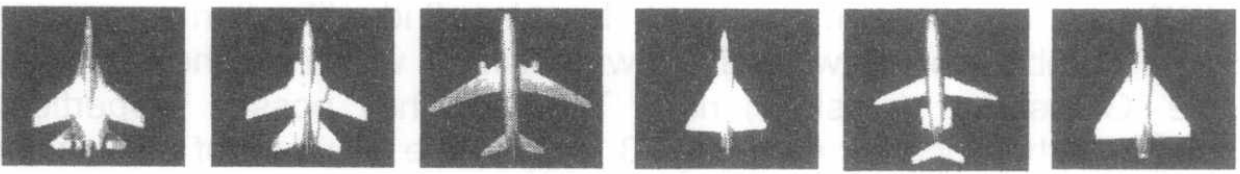

Fig. 1. From left to right are shown aircraft images after aspect of ratio correction.

The following steps were taken to obtain the normalized patterns:

1. At first the aspect ratio was carried out and the input patterns were resized to $50 \times 50$ pixels using bilinear interpolation.

2. Hotelling transform [6] was exploited to align the input image to positive $x$ axis in the image plane coordinate system.

3. Smoothing was performed using a median filter of size $3 \times 3$ pixels, which yield 'image1'.

4. A binary image was obtained by thresholding method, by setting all pixels greater than the predefined threshold to 255 and the others are set to 0 . This image is named 'image2'.

5. The aircraft image, named 'image3' was obtained using the clipping algorithm

$$
\begin{array}{r}
\boldsymbol{I F} \text { (image2=255) } \boldsymbol{T H} \boldsymbol{E N} \text { image3 }=\text { image1 } \\
\boldsymbol{E} \boldsymbol{L} \boldsymbol{S} \boldsymbol{E} \text { image3 }=\text { image } 2 .
\end{array}
$$

6. The clipping object was moved to a new position such that the coordinate of the object mean is $(25,25)$-pixel location.

The above steps were done experimentally using MATALB ${ }^{\circledR}$ and an example of aircraft image with different processing are shown in Fig. 2.
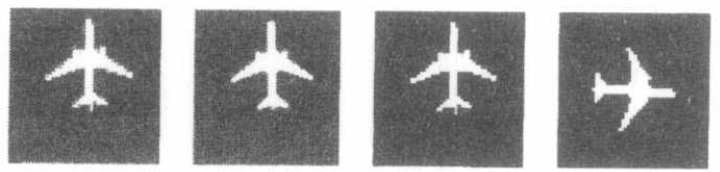

Fig. 2. From left to right be shown an example of the resized, smoothed, clipped, and normalized input pattern. 
III THE AIRCRAFT NEURAL MODEL

The neural network models divided mainly into two categories: (1) current networks or feed forward; (2) recurrent networks or feedback networks. The Hopfield net is considered as a good example for the recurrent networks. The response of such networks is dynamic since this output feedback to modify the input. However, the stability could be problem for this network. On the other hand, the lack of feedback path in the counter propagation models or feedforward network ensures that such networks are always stable.

The CPN was exploited in this work for aircraft identification purpose. The CPN is a combination of two well-known networks which are the Kohonen net and the Grossberg (outstare) net. Together they posses properties not available in either one alone. Fig. 3 shows the structure of CPN including Kohonen's layer and Grossberg layer. The neurons in layer zero act as distributors to Kohonen's layer and perform no computation.

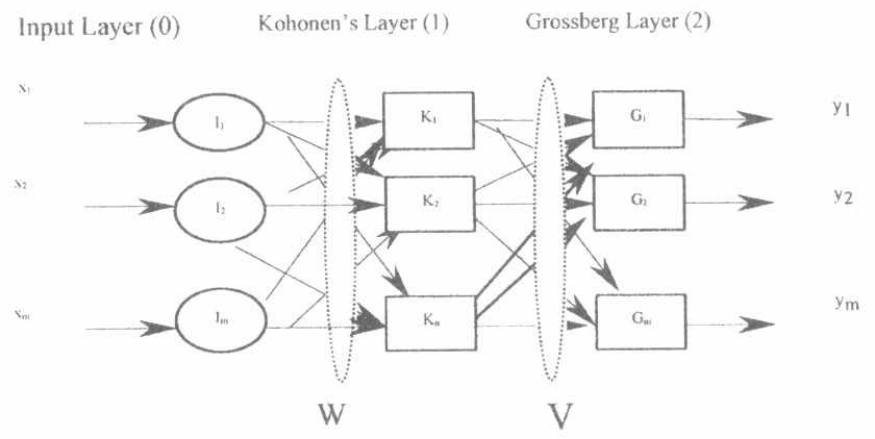

Fig. 3. Structure of aircraft neural network.

\section{Kohonen's layer}

The Kohonen's layer functions in a winner take-all manner; that is, for a given input vector, one and only one Kohonen's neuron outputs a logical one and all others output a zero. As shown in Fig. 3, each neuron in layer zero $x_{i}$ connects to every neuron in Kohonen's layer or layer-1 through a separate weight $w_{i j}$, Where $i=1,2, \ldots \ldots, n$ and $j=1,2, \ldots \ldots, m$. The Kohonen's neuron output $K_{i}$ is simply the summation of the weight inputs and can be defined in matrix form as:

$$
\begin{aligned}
& {\left[\begin{array}{l}
K_{1} \\
K_{2} \\
K_{n}
\end{array}\right]=\left[\begin{array}{lll}
w_{11} & w_{12} & w_{1 m} \\
w_{21} & w_{22} & w_{2 m} \\
& & \\
w_{n 1} & w_{n 2} & w_{n m}
\end{array}\right]\left[\begin{array}{l}
x_{1} \\
x_{2} \\
\\
x_{m}
\end{array}\right]} \\
& K=W X
\end{aligned}
$$


Where $W$ is the weight matrix after training. The Kohonen's neuron $K_{i}$ with the largest output value should be the "winner" and its value is set to one, and all others are set to zero. In other words, the activation function of Kohonen's neuron is represented by a step function. The learning approach of this net is a self-organizing algorithm that operates in the unsupervised mode. The training method can be described as:

1. Initialize the weight matrix $W$ of order nxm to random values.

2. For each class, the input pattern is represented by vector of $m$ size elements.

3. Normalizing each input vector $X_{i}$ before applying to the Kohonen's layer by dividing each element by its vector norm.

4. Adjust the weight vector such that

$$
W_{i}=W_{i-1}+\alpha\left(X_{i}-W_{i-1}\right)
$$

Where $\alpha$ is a training rate coefficient that usually $\leq 0.7$. It is apparent from equation (3) that the training process actually selects the Kohonen's neuron $K_{i}$ whose weight vector is most similar to the input vector.

5. The above steps are repeated to each class $i$ to calculate the training weight matrix.

\section{Grossberg layer}

The Grossberg layer functions are similar to Kohonen's layer except only one component of the input vector is nonzero and the others are zeros. In other words, the only item of each Grossberg neuron outputs the value of the weight that connects it to the single Kohonen's neuron marked 1. The training algorithm of this layer can be simply defined as:

1. Initialize the weight matrix of Grossberg layer $V$ of order $m x n$ to zero values.

2. The desired output vector is $Y_{i}$ of length $m$ represents a separate class.

3. Let the response of each Kohonen's neuron $K_{i}$ is set to 1 and other components are set to zero; then the training weighting vector $V_{i}$ to each output is defined as

$$
\begin{aligned}
& \Delta V=\beta\left(Y_{i}-V_{i-1}\right) K_{i}, \text { and } \\
& V_{i}=V_{i-1}+\Delta V
\end{aligned}
$$

Where $\beta$ is the learning coefficient and set to about 0.1 and is gradually reduced as training progress.

4. The above steps are repeated for all Kohonen's neurons to calculate the training weight matrix of Grossberg layer of order $m \times n$. 
Proceedings of the $\mathbf{2}^{\text {nd }}$ ICEENG Conference, 23-25 Nov 1999

\begin{tabular}{|l|l|}
\hline CE-6 & 7 \\
\hline
\end{tabular}

\section{CONCLUSIONS}

Neural networks have been used for image pattern recognition. In this paper, the CPN is exploited as an alternative method for aircraft identification. It is known that one of the disadvantages of neural network is that it does need a large amount of data and computation during learning stage. However in this work, only five version images of each class were used through the training phase.

It is difficult for a neural network to discriminate translated, scaled, and rotated objects. By using the bilinear interpolation and the principal component analysis, the presented aircraft network is invariant to location, dilation, and rotation. The neural network goes into failure, if the lighting condition is changed even with the same object, while in this work the presented neural network succeeded to recognize different gray scale images of the same object.

Finally, it was found that ANN model succeeded to recognize all input images when it was tested using 2058 images, some of them are partially incomplete, noisy and geometrically distorted.

\section{REFERENCES}

[1] Yen-Hao tseng, Jenq-Neng Hwang, and Florence H.Sheehan, "ThreeDimensional Object Representation and Invariant Recognition Using Continuous Distance Transform Neural Networks," IEEE Transaction on Neural Network, Vol. 8, PP. 141- 147, January (1997).

[2] Jianchang Mao and Anil K. Jain, Fellow, "Artificial Neural Networks for Feature Extraction and Multivariate Data Projection," IEEE Transaction on Neural Network, Vol. 6, PP. 296-316, March (1995).

[3] A. Ravichandran and B. Yegnanarayana, "Studies on Object Recognition From Degraded Images Using Neural Networks," IEE Transaction on Neural Network, Vol. 8, PP. 481-488, (1995).

[4] S. Caselli, E. Faldella, B. Fringuelli, L. Rosi, " A Neural Approach to Robotic Haptic Recognition of 3-D Objects Based on a Kohonen Self-Organizing Feature Map," IEEE Transaction On Neural Network, PP- 835-840, (1994).

[5] Stavros J. Perantonis and Paulo J. G. Lisboa, "Traslation, Rotation, and Scale Invariant Pattern Recognition by High-Order Neural Networks and Moment Classifiers," IEEE Transaction On Neural Network, Vol. 3 PP. 241251, (1992).

[6] A. A. Somaie, A. Badr and T. Salah, "Aircraft Recognition Using Eigenvector Technique", Proceedings of the sixteenth National Radio Science Conference, NRSC'99, Cairo, PP. C29-1: 9, February (1999). 
